tion; at any rate it offers much in attracting attention to accurate pelvimetry which will exercise caution in prognosis, and facilitate the progress toward scientific obstetrics.

1. Creating no necessity for premature artificial confinement.

2. Giving up all application of forceps and of all operations implicating the force of the head against the bony resistance of the pelvis (when it is developed or remains in the superior strait, in the excavation or in the inferior strait).

3. The absolute abrogation of embryotomy upon the living baby.

4. The enlargement of the size of the pelvis (by symphysiotomy, pubiotomy, ischio-pubiotomy, coccyotomy) in all the cases where the osseous resistance is not overcome by the uterine contractions, the position of the head being well determined, and together with its size, where the calculation demonstrates that when section of the pelvis is made, its enlargement will not be under 7 c.m., allowing the passage of head of the fetus at term.

5 . Where absolute contraction of the pelvis is present (under 7 c.m.) the section serves for the Porro operation.

\section{SUSPENSIO UTERI-THE PROPER METHOD OF PERFORMING IT, AND ITS} RESULTS.

Read in the Section on Obstetrics and Diseases of Women, at the Forty sixth Annual Meeting of the American Medical Association, at Baltimore, Md., May 7-10, 1895.

BY HOWARD A. KELLY, MI.D.

BALTIMORE, MD.

(ABSTRACT.)

Dr. Kelly refused to accept the name of ventrofixation or hysteropexy. The uterus is not fixed. He prefers the name of suspensio-uteri as more accurately describing the condition. In the past five years he has performed this operation one hundred and seventy times and thirty-seven times in the past year. The indications for the operation are extreme local discomfort associated with uterine displacements, and neurasthenia, with backache and headache. In the first class of cases, with local symptoms but no general symptoms, the operation is plain. In the last series of cases it is difficult to say just when the operation is indicated. The most brilliant cures, however, have been in this class of cases. He is willing to take the chances and fail in four cases in order to get one good cure. Of the 132 cases reported, 90 were married; and of the 78 per cent. had borne children, and 14 of them had had miscarriages. Not one died or showed bad symptoms. Transient mania has occurred in three cases, pneumonia in one case, and stitch-abscess in three cases. Cystitis and frequent urination had occurred in four cases only, and had been but transient.

The operation is simple. The pelvis should be slightly elevated, and a small incision made just above the symphysis pubis about one and a half or two inches in length. The peritoneum is incised and drawn out with forceps. Two fingers are inserted to the fundus, and the uterus hooked up. Adhesions are stripped off with the fingers or cut with scissors or knife, and the uterus anteflexed. The abdominal wall is lifted on the left side until the peritoneum can be seen for one inch away from the line of the incision. A needle is then carried through the peri- toneum, but not entering the muscular tissue, and then through the posterior uterine wall just below the fundus, taking in about one-fourth of an inch in length, and extending about one-eighth of an inch in depth. The suture is then carried to the opposite side of the abdominal wall. Another stitch is passed. just above the first, near the incision, and inserted into the uterus below the other, and then carried back to the opposite side of the abdominal wall. This increases the anteflexion. A third suture may be inserted. The peritoneum is then closed by a continuous suture; then the fascia is closed and then the skin incision. The distance between the uterus and anterior abdominal wall is about one or one and one-half inches. The organ is attached by a strong fibrous cord which cuntains the sutures close to the abdominal wall. Pregnancy is not seriously interfered with. In only one case, and that after two years, did the uterus drop back.

\section{THE TREATMENT OF HEMORRHAGIC CON- DITIONS OF THE UTERUS BY ZINC- AMALGAM ELECTROLYSIS.}

Read in the Section on Obstetrics and Diseases of Women, at the Forty . sixth Annual Meeting of the American Medical Association, at Baltimore, Md., May 7-10. 3895 .

BY G. BETTON MASSEY, M.D.

$$
\text { PHILADELPHIA. }
$$

The use of a soluble metallic electrode as the positive pole of a galvanic current is specially avoided in the usual applications to the uterine and other mucous cavities whenever the pure action of the electricity alone is desired. To avoid this disintegration of the electrode and the consequent local action of the nascent metallic salt produced by its erosion, it has been necessary to employ non-oxidizable electrodes with this pole; platinum and carbon being the only practicable materials. It has however, been more recently discovered that the nascent salts produced by the corrodible electrodes have a distinct value in certain cases, particularly those in which a local alterative and antiseptic action is desirable. The salts produced by this erosion of a base metal anode are oxychlorids, produced by union of the free oxygen, and chlorin developed by the electrical action, and being in a nascent condition are particularly active. Copper, zinc and iron have been the substances employed by Onimus, Gautier (who has especially studied the method), Morton, Goelet, Cleaves and others, under the designation of interstitial electrolysis, metallic electrolysis and cupric electrolysis.

My own work has been almost entirely with zinc, and was originally directed exclusively toward the treatment of incipient malignant conditions, in which the nascent zinc salt has been a most valuable adjuvant to the lethal action of a concentrated current. Owing to the practical difficulty that at times resulted from the adhesion of the electrode to the surface after a prolonged application, and also on account of the roughened surface rapidly attained by the electrode, I was led to amalgamate the zinc freely with mercury before using, and am convinced that the expedient is a valuable one. Not only does this keep the zinc surface always smooth, lubricated and nonadhesive, but a new value is attained in the use of a nascent oxychlorid of mercury in addition to the oxychlorid of zinc and a far more efficient alterative and antiseptic action results. 
The additional effect given by this process to the galvanic treatment of hemorrhagic endometritis is shown in the following case histories:

Case 1.-Mrs. J. F., aged 54, was referred to me by Dr. H. W. Elmer, of Bridgeton, N.J., April 17, 1894. For three or four years she had been suffering from an almost continuous hemorrhage, resulting in great prostration. Shortly after the beginning of the hemorrhages, a tumor of the uterus was discovered, which was the size of a large fist when I first saw ber. She was placed under the Apostoli treatment which produced considerable improvement in the hemorrhages but did not even after six months' occasional treatment entirely correct the hemorrhagic tendency. Fearing that the case was a malignant one in spite of the absence of marked pain, I decided to substitute an amalgamated zinc electrode for the carbon one previously employed. It was found that the applications with this electrode were better borne, enabling them to be used more frequently. Six applications were made in a period of two weeks in December last, completely checking the dribbling, and since then there has been no real hemorrhage and the patient has been restored to health. The tumor was materially reduced in size.

Case 2.-Miss C. F. H., aged 31 , had been under treatment for some time for a uterine fibroid of large dimensions, accompanied by profuse and exhausting menorrhagia. Under the ordinary Apostoli treatment the tumor had been reduced somewhat and the menorrhagia lessened, but she still had periods that were too profuse for her impaired strength. In January and February of the present year the electrode for intra-uterine treatment was changed from carbon to amalgamated zinc, the same current strength, 150 milliamperes, being employed. All periods subsequent to these applications have been absolutely normal.

Case 3.-Mrs. F., aged 47, had also been under treatment for a hemorrhagic fibroid of smaller size, with a similar history of decrease and partial control of the hemorrhagic feature. The metal of the intra-uterine anode was changed from platinum to amalgamated zinc about the same time, a current strength of 60 being employed. Up to this time her periods had always anticipated by several days, notwithstanding her age, but the first period after the changed treatment was postponed a week, and they have been delajed and normal in amount since.

1636 Walnut Street.

\section{A ËRO.URETHROSCOPY.}

Read in the Section on Surgery and Anatomy at the Forty-sixth Annual Meeting of the American Medical Association, at Baltimore, Md., May 7-10, 1895 .

BY WILIIAM K. OTIS, M.D.

ATTENDING SURGEON TO THE CITX HOSPITAL. NEW YORK; TO ST. MARK' HOSPITAL, NEW YORK, ETC., ETC. NEW YORK.

Notwithstanding the very great improvements made in urethroscopic instruments during the past few years, the urethroscopic field, in point of size, is still far from being entirely satisfactory. Since the invention of the original illuminating apparatus, little if any change has been made in urethroscopic tubes, though they have borne various names and been constructed of many different materials.

Glass from its transparency seemed especially adapted to this purpose, but the reflections from its surface and the changes produced by pressure on the mucous membrane, together with the danger of fracture if used in the deeper urethra, reuder it practically valueless. The discovery in 1870 of the great underestimation of the normal urethral caliber permitted the use of much larger tubes, with a corresponding increase in the size of the field; but the practical urethroscopic tube remained and still remains a simple straight tube.

In the year 1887 the late Geza von Antal, of BudaPesth, in the endeavor to extend the urethroscopic field, evolved the ingenious idea of distending the arethra with atmospheric air. $\mathrm{He}$ invented an instrument for this purpose which was eminently successful, consisting of a tube with a well-fitting obturator, $6 \mathrm{~cm}$. long, with a concave metal shield at its proximal end which fitted over the glans penis, the urethral orifice being drawn upward against this so as to prevent the escape of air. After the introduction of the tube an ocular portion, rendered airtight by a glass plate, was attached by a sliding joint. By means of a small tube entering the ocular portion at right angles, the urethra was inflated with a rubber bulb and an inspection of its mucous membrane obtained by a beam of light reflected from a head mirror ${ }^{1}$.

This instrument, however, failed to attract much attention and the method met with but little favor among urethroscopists in general.

The examination of the inflated urethra was taken up, independently of von Antal's work, in this direction, by Heule of New York (1890) who devised a simpler but equally effective instrument for accomplishing the same results.

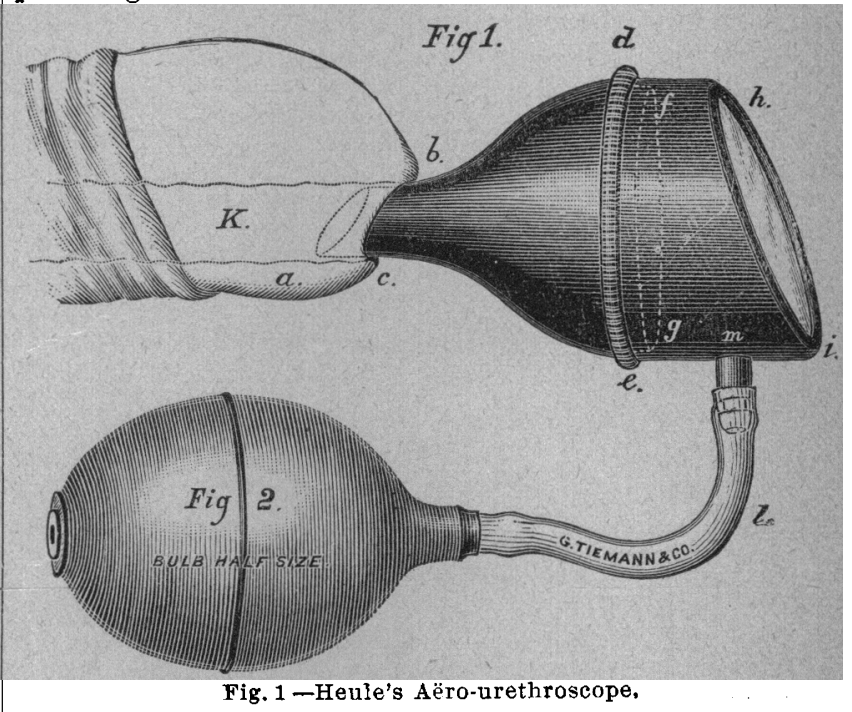

Heule's instrument consists of two parts, viz., "the speculum, $a, b, d, e$, and the cover, $f, g, h, i$. The speculum is made of rubber, glass or metal, shaped like a truncated cone, with the end $a, b$, cut obliquely to facilitate its introduction into the urethra without the aid of a stylet. At the other or proximal end is a flange, $d, e$, which is useful in separating the cover from the speculum. Into this proximal end is closely fitted the removable cover, $f, g, h, i$, having at $h, i$, a transparent glass crystal, fitted air-tight, and at $m$ an attachment for a rubber tube with bulb (Fig. 2) for forcing atmospheric air into the endoscope.

"To prevent the glare of reflected light, the glass crystal at $h, i$, is placed at an obtuse angle to the line of vision, and the inside of the instrument is blackened. The specula at $a, b, c$, are made of various sizes to fit small and large openings, but at the proximal end, $d, e$, are all adapted to one cover, $f, g, h, i$.

"In using this instrument the flaccid penis is grasped between the ring and middle fingers of the left hand, the lips of the meatus being opened with the index finger and thumb of the same hand. The endoscope held in the right hand by the flange, $d, e$, can now be readily introduced into the urethra by means of the oblique end, $a, b, c$, until the thicker portion of the speculum, near $d, e$, fills the opening. Air is now 